DOI: https://doi.org/10.46296/gt.v4i8edespnov.0022

\title{
PERFIL EPIDEMIOLÓGICO DE LAS INFECCIONES RESPIRATORIAS INTRAHOSPITALARIAS. HOSPITAL DR. VERDI CEVALLOS BALDA. ECUADOR.
}

\section{EPIDEMIOLOGICAL PROFILE OF INTRAHOSPITAL RESPIRATORY INFECTIONS. HOSPITAL DR. VERDI CEVALLOS SHELF. ECUADOR.}

\author{
Rosado-Moreira John Alfredo ${ }^{\text {; }}$ Intriago-Cedeño María Cecibel ${ }^{2}$; \\ Padilla-Urrea Carmen Marlene ${ }^{3}$ \\ ${ }^{1}$ Universidad Técnica de Manabí, UTM. Portoviejo, Ecuador. Correo: tonyalfredo- \\ 2013@outlook.com. ORCID ID: https://orcid.org/0000-0002-2201-111X \\ ${ }^{2}$ Universidad Técnica de Manabí, UTM. Portoviejo, Ecuador. ORCID ID: https://orcid.org/0000- \\ 0003-4965-4315 \\ ${ }^{3}$ Universidad Técnica de Manabí, UTM. Portoviejo, Ecuador. ORCID ID: https://orcid.org/0000- \\ 0002-2711-9154
}

\begin{abstract}
Resumen
Introducción: Es conocido a nivel mundial que las infecciones intrahospitalarias constituyen un problema serio en salud pública, particularmente cuando la mayoría de los casos que ocurren se deben a la implementación de estrategias no recomendadas y malas prácticas generales. Objetivo general: determinar el perfil epidemiológico de las infecciones respiratorias intrahospitalarias en el Hospital General Dr. Verdi Cevallos Balda. Método: Se realizo un estudio descriptivo retrospectivo y transversal, el período de estudio incluyo el año 2019. El universo estuvo constituido por los 28 pacientes diagnosticados con infecciones respiratorias intrahospitalarias registrados en el Subsistema de vigilancia activa de las infecciones asociadas a la salud (IAAS). Los datos fueron recolectados de las historias clínicas individuales a través de una ficha de recolección de datos de acuerdo a los factores que conforman la cadena epidemiológica: microorganismo causal, huésped susceptible y tratamiento recibido. Resultados: El staphylococcus aureus fue el microorganismo de mayor repercusión (35,7\%). En las características del huésped susceptible predominaron la edad mayor 60 años $(57,1 \%)$ y en las enfermedades subyacentes la diabetes mellitus ( $42,8 \%)$, el sexo no fue relevante. El tratamiento inicial como final los betalactámicos fueron los de mayor utilización con $68 \%$ y $71,4 \%$ respectivamente, la aplicación de sonda nasogástrica $(60,7 \%)$ y la ventilación mecánica $(39,2$ $\%)$ fueron los factores de riesgo más representativos. Se evidencio además que la estancia mayor a 15 días aumento su incidencia. Conclusiones: Se puede concluir que el perfil epidemiológico de las infecciones respiratorias intrahospitalarias está determinado por factores que incidieron directamente sobre los cuales se debe incidir para su prevención.
\end{abstract}

Palabras clave: Infecciones hospitalarias; Infecciones nosocomiales; Infecciones del sistema respiratorio; Ventilación mecánica; Neumonía.

\section{Abstract}

Introduction: It is known worldwide that intrahospital infections constitute a serious problem in public health, particularly when the majority of cases occur in the implementation of nonrecommended strategies and general bad practices. General objective: to determine the epidemiological profile of intrahospital respiratory infections at the Dr. Verdi Cevallos Balda General Hospital. Method: A retrospective and cross-sectional descriptive study was carried out;

Información del manuscrito:

Fecha de recepción: 17 de septiembre de 2021.

Fecha de aceptación: 29 de octubre de 2021.

Fecha de publicación: 04 de noviembre de 2021. 
the study period included the year 2019. The universe consisted of the 28 patients diagnosed with intrahospital respiratory infections registered in the Active Surveillance Subsystem of Health Associated Infections (IAAS). Data were collected from individual medical records through a data collection form according to the factors that make up the epidemiological chain: causal microorganism, susceptible host, and treatment received. Results: Staphylococcus aureus was the microorganism with the greatest repercussion (35.7\%). In the characteristics of the susceptible host, age over 60 years $(57.1 \%)$ predominated and in underlying diseases, diabetes mellitus (42.8\%), sex was not relevant. Initial and final treatment, beta-lactams were the most widely used with $68 \%$ and $71.4 \%$ respectively, the application of a nasogastric tube $(60.7 \%)$ and mechanical ventilation (39.2\%) were the most representative risk factors. It was also evidenced that a stay longer than 15 days increased its incidence. Conclusions: It can be concluded that the epidemiological profile of intrahospital respiratory infections is determined by factors that had a direct influence on which should be influenced for their prevention.

Keywords: Hospital infections; Nosocomial infections; Respiratory system infections; Mechanical ventilation; Pneumonia.

\section{Introducción}

Es conocido a nivel mundial que las infecciones intrahospitalarias constituyen un problema serio en salud pública, particularmente cuando la mayoría de casos que ocurren se deben a la implementación de estrategias no recomendadas y malas prácticas generales. El contagio hospitalario se comenzó a tener en cuenta sólo a partir de la mitad del siglo XIX, y con no pocas discusiones en el entorno médico de la época, ya que, para los galenos de la época, era inadmisible pensar en que el médico, el sanador de enfermos era también propagador de enfermedades. $(1,2)$

Una encuesta de prevalencia realizada bajo los auspicios de la Organización Mundial de la Salud en
55 hospitales de 14 países representativos de 4 Regiones de la OMS (Europa, el Mediterráneo Oriental, el Asia Sudoriental y el Pacifico Occidental) mostró que un promedio de $8.7 \%$ de los pacientes hospitalizados presenta infecciones nosocomiales. (3)

Se estima que más de 1,4 millones de personas en todo el mundo sufren infecciones contraídas en los hospitales, siendo las regiones de mayor incidencia Europa y Asia Sudoriental. Entre un cinco y un $10 \%$ de los pacientes hospitalizados en Europa y Norteamérica son afectados por las infecciones asociadas a la asistencia sanitaria. En el caso de las regiones de Asia, América Latina y África subsahariana esta cifra sobrepasa el 
$40 \%$ de los casos de hospitalizaciones. (4)

Actualmente se estima que las infecciones asociadas a la atención en salud (IAAS) más frecuente es la infección urinaria producida por el uso de catéteres o sondas de vía urinaria $(40 \%)$, en segundo lugar, se encuentra la infección de heridas quirúrgicas la cual representan hasta un $25 \%$ de las IAAS, las infecciones respiratorias se estima que se presentan de un 15 a $20 \%$. (5)

En Estados Unidos de la Red Nacional de Seguridad Sanitaria (NHSN) examinó los tipos más frecuentes de las infecciones nosocomiales en unidades de cuidados intensivos debido a bacilos gramnegativos, éstas representaron la siguiente proporción neumonía asociada con la ventilación: $8.4 \%$, infección del torrente sanguíneo relacionada con catéter central: $2.2 \%$, infección de las vías urinarias vinculada con catéter: $1.2 \%$, infección del sitio quirúrgico: $0.6 \%$.(6) Siendo las respiratorias las de mayor incidencia.

En el estudio de prevalencia publicado en el Informe de vigilancia IASS en Chile se evidencia que las neumonías asociadas a ventilación mecánica invasiva (NAVM) son la séptima infección más frecuente en chile en 2018 , donde el $3,28 \%$ de los pacientes recibieron ventilación mecánica, especialmente en los hospitales de mayor complejidad, donde esta prevalencia subió al $3,29 \%$, reportando un total de 1.168 NAVM representando el $96,3 \%$ de los pacientes con ventilación mecánica. (7)

El Instituto Nacional de Angiología y Cirugía Vascular en Cuba realizo un estudio sobre las Infecciones asociadas a la atención sanitaria donde se evidencio que segundo lugar en frecuencia (25\%) se encuentran las infecciones respiratorias, sobre todo las bronconeumonías, donde los pacientes con estadías prolongadas resultan los más vulnerables, así como aquellos que tienen riesgos que los predisponen como son los fumadores y ancianos, por lo que tienen cierta disposición a este tipo de infección, que debe prevenirse en primer lugar y buscarse activamente en todos los pacientes quirúrgicos (8).

En Ecuador, según la información del Sistema de Vigilancia de 
Enfermedades (SIVE) y Eventos de Notificación Obligatoria del Subproceso de Epidemiología del Ministerio de Salud Pública, las IASS de mayor frecuencia en estudio de incidencia durante los años 2017 y 2018 fueron las neumonías asociadas a la ventilación mecánica con una densidad por años en estudio de 8,70 y 8,40 respectivamente en los servicios de cuidados intensivos de adultos y pediátricos y ocupando el segundo lugar en los servicios de cuidados intensivos e intermedios neonatales con una densidad de incidencia de 3,96 en 2017 y 4,03 en 2018. (9)

Es importante agregar que a partir del año 2016 se empezó a fortalecer este subsistema de vigilancia epidemiológica con la participación de 13 unidades centinela a nivel nacional y actualmente cuenta con 50 hospitales siendo el Hospital Verdi Cevallos Balda una de ellas. Este subsistema ha establecido la vigilancia de trece eventos relacionados a las IAAS, enmarcados en tres componentes principales, y al realizar el comparativo con países vecinos el Ecuador presenta tasas elevadas en la neumonía asociada a ventilación mecánica con una densidad de incidencia de 8.40/1000 días de exposición al riesgo de infección, mucho mayor que Perú $(7,52)$ y Chile con 6,06 (adultos) 2.56 (pediátricos) en servicios de cuidados intensivos adultos y pediátricos. (10)

Al ser el 2018 el segundo año que se cuenta con datos a nivel Nacional, no se puede definir aún un canal epidemiológico, pero se evidencia que los eventos relacionados a los dispositivos médicos invasivos ocupan los primeros puestos en densidad de incidencia como son la Neumonía asociada a ventilación mecánica (NAV) y la Infección del tracto urinario asociado a catéter urinario permanente (ITS /CVC). Como se mencionó, al no contar con datos a nivel nacional previos al inicio de la puesta en práctica del subsistema de vigilancia epidemiológica, no existen datos de estimación referencial al canal epidemiológico. Sin embargo, con respecto al número de casos y densidad de incidencia de IAAS del componente UCl Adulto y pediátrico por tipología de las unidades centinela en el año 2018 las NAV es el evento con mayor número de casos reportados en este 
componente, siendo los hospitales generales los que reportan una mayor densidad de incidencia (11.18/1000 días de exposición).9, 10

Según el ministerio de salud pública ecuatoriano hay estadísticas que oscila entre el $24 \%$ al $75 \%$ de mortalidad anual en los establecimientos hospitalarios, por causa de la neumonía asociada a ventilación mecánica en el periodo 2018-2019 respectivamente. 10

Por todo lo anteriormente expuesto, el objetivo de la presente investigación es determinar el perfil epidemiológico de las infecciones respiratorias intrahospitalarias en el Hospital General Dr. Verdi Cevallos Balda durante el año 2019 lo que permitirá identificar los factores que inciden directamente en su ocurrencia y poder controlarlos para su prevención.

\section{Métodos}

Se realizó un estudio de tipo descriptivo retrospectivo y transversal, el período de estudio incluye enero a diciembre de 2019.

El universo estuvo constituido por los 28 pacientes diagnosticados con infecciones

respiratorias intrahospitalarias registrados en el Subsistema de vigilancia activa de las infecciones asociadas a la salud (IAAS).

Los datos fueron recolectados de las historias clínicas individuales a través de una ficha de recolección de datos (anexo-1) de acuerdo a los factores que conforman la cadena epidemiológica tales como: microorganismo causal, huésped susceptible y tratamiento recibido.

En cuanto a las diferentes variables se detallarán dimensiones a ser analizadas tales como:

- Agente causal: será determinado por los agentes patógenos presentes y aislados por cultivo.

- Huésped susceptible: Edad, sexo biológico y enfermedades subyacentes.

- Tratamiento recibido:

Tratamientos inmunosupresivos, Antimicrobianos de inicio y fin del tratamiento y mecanismos invasivos aplicados.

Para describir el comportamiento de las variables se analizaron de forma univariada con los métodos de 
estadística descriptiva frecuencias absolutas y porcentajes.

La investigación contó con la aprobación del Comité de Ética para la Investigación de la Facultad de Ciencias de la Salud de la Universidad Técnica de Manabí, así como con la autorización de la unidad (hospital seleccionado). Se cumplieron las normas éticas establecidas en la Declaración de Helsinki de 1975 revisadas en octubre del 2000.

\section{Resultados}

El staphylococcus aureus fue el microorganismo de mayor incidencia durante el diagnostico etiológico de las infecciones respiratorias intrahospitalarias con 10 casos para un $35,7 \%$. Vale destacar que en el $25 \%$ de los pacientes no se aisló el germen causal de la infección, donde en la mayoría de los casos fueron reingresos lo cual habla a favor de deficiencias en la atención, tratando la infección de manera empírica (Tabla 1).

Gráfico 1. Microorganismos causales más frecuentes aislados en cultivos.

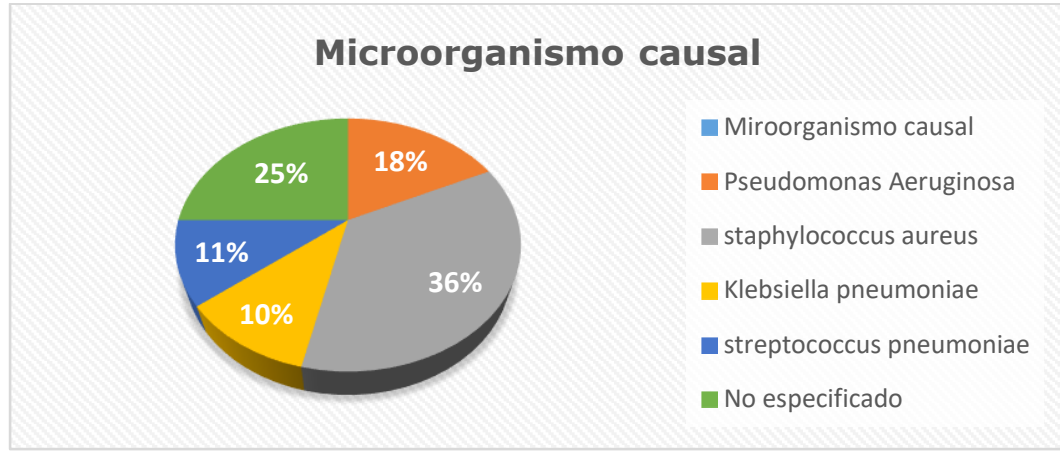

$N$ - Total de pacientes con IASS

Fuente: Ficha de recolección de datos.

Dentro de la cadena epidemiológica de las infecciones intrahospitalarias se encuentran las características del hospedero susceptible donde la edad, el sexo y el estado de salud juegan un papel determinante (Grafico- 1). En cuanto a la edad podemos decir que en la medida que avanzamos en edad aumentan las posibilidades de la aparición de estas entidades patológicas siendo los pacientes mayores de 60 años los más afectados en un $57,1 \%$. Al analizar el sexo se evidencia que no es una característica relevante puesto que al ser una enfermedad infecciosa puede atacar a ambos de la misma manera, aunque existió mayor afectación en el sexo masculino en un $64,2 \%$. De acuerdo 
a los datos obtenidos referentes a las enfermedades subyacentes la más frecuente fue la diabetes mellitus con un $42,8 \%$, vele destacar que a diferencia de otros estudios revisados el mayor por ciento de pacientes no poseía antecedentes patológicos en un 32,1\%.

Tabla 1. Distribución según características del hospedero susceptible.

\begin{tabular}{|c|c|c|c|}
\hline \multirow[t]{2}{*}{ Características } & \multicolumn{3}{|c|}{ Casos de infecciones respiratorias intrahospitalaria. } \\
\hline & FA & $\mathrm{N}=28^{*}$ & $\%$ \\
\hline \multicolumn{4}{|l|}{ Edad } \\
\hline$<20$ años & & 7 & 25,0 \\
\hline $21-40$ años & & 3 & 10,7 \\
\hline $41-60$ años & & 2 & 7,1 \\
\hline Más 60 años & & 16 & 57,1 \\
\hline \multicolumn{4}{|l|}{ Sexo } \\
\hline Femenino & & 10 & 35,7 \\
\hline Masculino & & 18 & 64,2 \\
\hline \multicolumn{4}{|c|}{ Enfermedades Subyacentes } \\
\hline Asma bronquial & & 3 & 10,7 \\
\hline Hipertensión arterial & & 4 & 14,2 \\
\hline Diabetes mellitus & & 12 & 42,8 \\
\hline Ninguna enfermedad & & 9 & 32,1 \\
\hline
\end{tabular}

Fuente: Ficha de recolección de datos.

Del total de pacientes con infecciones intrahospitalarias el 100 $\%$ no tuvo tratamientos inmunosupresores. En relación al tratamiento antimicrobiano en el tratamiento inicial como final los betalactámicos fueron los de mayor utilización con $68 \%$ y $71,4 \%$ respectivamente, se pudo comprobar que no existió un adecuado cumplimiento de los protocolos de uso de antimicrobianos.

Tabla 2. Factores de riesgo referentes al tratamiento recibido.

\begin{tabular}{|c|c|c|c|c|}
\hline \multirow{2}{*}{$\begin{array}{l}\text { Tratamiento } \\
\text { recibido }\end{array}$} & \multicolumn{2}{|c|}{ Antimicrobiano inicial } & \multicolumn{2}{|c|}{ Antimicrobiano final } \\
\hline & FA & $\%$ & FA & $\%$ \\
\hline Betalactámicos & 19 & 68,0 & 20 & 71,4 \\
\hline Macrólidos & 1 & 3,5 & 0 & 0,0 \\
\hline Quinolonas & 2 & 7,1 & 3 & 10,7 \\
\hline Glucopéptidos & 2 & 7,1 & 2 & 7,1 \\
\hline Aminoglucósidos & 1 & 3,5 & 3 & 10,7 \\
\hline Lincosamidas & 3 & 10,7 & 0 & 0,0 \\
\hline
\end{tabular}

Fuente: Ficha de recolección de datos. 
En cuanto a los factores de riesgo asociados a mecanismos invasivos se pudo constatar en la mayoría de los casos diagnosticados con infecciones respiratorias intrahospitalarias fueron aplicados mecanismos invasivos en un tiempo de 7 a 15 días y más. Donde la aplicación de sonda nasogástrica $(60,7 \%)$ y la ventilación mecánica $(39,2 \%)$ fueron los factores de riesgo más representativos.

Tabla 3. Factores de riesgo según mecanismos invasivos aplicados.

\begin{tabular}{|c|c|c|c|c|c|c|}
\hline \multirow[t]{3}{*}{ Mecanismos invasivos } & \multicolumn{6}{|c|}{ Tiempo de aplicación } \\
\hline & \multicolumn{2}{|c|}{-7 DÍAS } & \multicolumn{2}{|c|}{ 7-15 DIAS } & \multicolumn{2}{|c|}{ MÁS DE 15 DÍAS } \\
\hline & FA & $\%$ & FA & $\%$ & FA & $\%$ \\
\hline Sonda vesical & 9 & 32,1 & 1 & 3,5 & 10 & 35,7 \\
\hline Sonda nasogástrica & 7 & 25 & 17 & 60,7 & 2 & 7,1 \\
\hline Abordaje venoso periférico & 3 & 10,7 & 9 & 32,1 & 4 & 14,2 \\
\hline Drenes & 0 & 0,0 & 7 & 25 & 2 & 7,1 \\
\hline Ventilación mecánica & 6 & 21,4 & 11 & 39,2 & 0 & 0,0 \\
\hline Otros* & 0 & 0,0 & 0 & 0 & 1 & 3,5 \\
\hline
\end{tabular}

*otros: Sondaje transpilórico.

Fuente: Ficha de recolección de datos.

Entre los factores relacionados con el medio ambiente hospitalario se pudo comprobar que la estancia hospitalaria (grafico-2) aumenta el riesgo de sufrir una infección, los pacientes que tuvieron una estancia mayor a 15 días fueron los más afectados $(42,8 \%)$.

Grafico 2. Estadía hospitalaria.

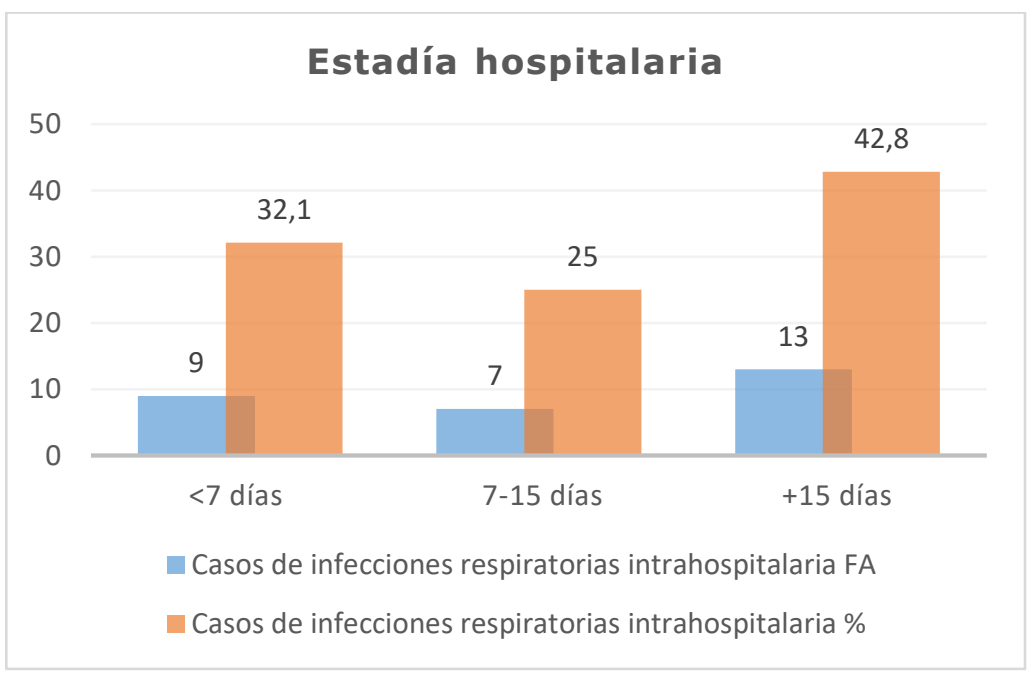

Fuente: Ficha de recolección de datos. 


\section{Discusión}

Establecer el perfil epidemiológico de las infecciones respiratorias intrahospitalarias es de gran importancia para diseñar estrategias de prevención. En la presente investigación se estableció que el staphylococcus aureus fue el microorganismo de mayor incidencia durante el diagnostico etiológico. Es a su vez, el antecedente de mortalidad de mayor recurrencia entre las infecciones nosocomiales, prioritariamente, las derivadas a Pseudomonas aeruginosa $y$ Staphylococcus aureus resistente a meticilina (MRSA) (10) que es un betalactámico muy usado en su tratamiento siendo en la presente investigación el medicamento de elección al inicio y final con que fueron tratados la mayoría de los pacientes. Al valorar la importancia de determinar el microorganismo que con mayor frecuencia causo dichas afecciones podemos indicar que no solo es relevante para el medio hospitalario, sino que también para el comunitario pues pueden ser transmitidos a la comunidad por los pacientes después del alta hospitalaria, por lo que si dichos microorganismos son multirresistentes, pueden causar enfermedad grave en los miembros de la comunidad, siendo difícil su tratamiento.

La resistencia de Staphylococcus aureus a los betalactámicos como la meticilina ocurre por la adquisición del gen mecA el cual codifica una proteína ligadora de penicilina "alternada" (PBP2a) que no permite la unión con los betalactámicos, esta PBP2a tiene la capacidad de sintetizar el peptidoglicano de forma simultánea. Un estudio en Lima demostró que el $50 \%$ de Staphylococcus aureus aislados de hemocultivos de varios hospitales de Lima en el periodo 2008 - 2009 fueron resistentes a meticilina (11). Sin embargo, en la presente investigación se demostró que dichos fármacos fueron la elección inicial y final de tratamiento como ya se declaró anteriormente, así como los glucopéptidos.

Los niveles segundo y tercero de la lista de la OMS -las categorías de prioridad alta y media- contienen las bacterias que exhiben una farmacorresistencia creciente dentro de ellos los aislados en el presente estudio como son, Staphylococcus aureus resistente a la meticilina, con 
sensibilidad intermedia y resistencia a la vancomicina, y la Shigella spp., resistente a las fluorquinolonas. Estas declaraciones se fundamentan en estudios publicados con nivel alto de evidencias y otros revisados hasta la actualidad. (12)

En el Ecuador la situación referente a lo anteriormente expuesto es casi igual a los demás países. Según el ministerio de salud pública entre el $24 \%$ al $75 \%$ de mortalidad anual en los establecimientos hospitalarios, fue debido a la neumonía asociada a ventilación mecánica en el periodo 2019, siendo las edades mayores de 60 años las más afectadas, lo cual corrobora la presente investigación y otras similares $(13,14)$. Otros estudios establecen que el $80 \%$ de los cuadros clínicos distinguidos por neumonía nosocomial o intrahospitalaria configuran en pacientes con vía aérea artificial. (15) Aunque el Hospital Dr. Verdi Ceballos Balda (miembro de la red de hospitales centinelas para el diagnóstico y notificación obligatoria de dichas infecciones) reporto solo 28 casos del total de pacientes hospitalizado y solo 17 de ellos fueron tributarios de ventilación mecánica.

En relación a las enfermedades subyacentes la diabetes mellitus fue la principal comorbilidad. Es conocido, que con el incremento de la edad es mayor la posibilidad de presentar alguna comorbilidad, tal es el caso de un estudio realizado por Chong YP, donde el $45,1 \%$ de los pacientes evidenció algún factor de riesgo, teniendo la mayor frecuencia las enfermedades crónicas no transmisibles, pero en ese caso fue la diabetes mellitus, quien ocupó el primer lugar, seguido de la HTA. $(16,14)$

Mientras más prologada es la ventilación mayor será el riesgo de complicaciones. Cuando los pacientes están expuestos mayor tiempo a la ventilación mecánica, el tubo endotraqueal interfiere en la barrera fisiológica del sistema respiratorio y se produce una inhibición de los mecanismos de limpieza (movimiento mucociliar y tos), lo cual facilita la microaspiración del contenido orofaríngeo, además de causar lesión tisular que posibilita la adherencia bacteriana. (17)

Otro factor que influye en la incidencia de infecciones 
respiratorias intrahospitalarias es el aumento de la colonización de la orofaringe y/o del estómago por microorganismos por lo que la colocación de la sonda nasogástrica puede incidir directamente. Por lo que descontaminación digestiva selectiva indica un método de prevención de la infección mediante la erradicación de la colonización de bacterias aeróbicas potencialmente patógenas desde la orofaringe, estómago e intestino. (18)

\section{Conclusiones}

Que el perfil epidemiológico de las infecciones respiratorias intrahospitalarias en el Hospital Dr. Verdi Cevallos Balda está determinado por factores que incidieron directamente. Quedando formada su cadena epidemiológica, como microorganismo causal el staphylococcus aureus, en cuanto a las características del huésped susceptible las edades mayores de 60 años y la presencia de enfermedades crónicas no trasmisibles como la diabetes mellitus, siendo el sexo no relevante, en el tratamiento recibido los betalactámicos fueron la elección inicial y final. En relación a los mecanismos invasivos aplicados la ventilación mecánica y el sondaje nasogástrico, y la estancia hospitalaria mayor de 15 días. Las acciones de prevención deben estar dirigidas a cada uno de los eslabones de dicha cadena.

\section{Bibliografía}

1. Pérez Abreu MR, Gomez Tejeda JJ, Crúz Diaz J, Diéguez Guach RA. Infecciones nosocomiales en la Unidad de Cuidados Intensivos del Hospital General de Gibara: 20132018. CCM [Internet]. 2021 [citado 16 Ago 2021]; 25(3):[aprox. 0 p.]. Disponible en:

http://www.revcocmed.sld.cu/i ndex.php/cocmed/article/view /3776

2. Baby $N$, Faust $A C$, Smith $T$, Sheperd LA, Knoll L, Goodman EL. La prueba de PCR nasal para Staphylococcus aureus resistente a la meticilina (MRSA) reduce la duración de la terapia dirigida contra MRSA en pacientes con sospecha de neumonía por MRSA.

Agentes antimicrobianos Chemother. 2017 [citado 16 Jul 2021]; 61 (4): 02432-02516. Disponible en:

https://journals.asm.org/doi/fu II/10.1128/AAC.02432-16 
3. Organización Mundial de la Salud.( OMS). Prevención de las infecciones nosocomiales. Guía Práctica. Segunda Edición.2018 [citado 2021 Jul 24].Disponible

en:https://www.paho.org/hq/in dex.php?option=com_docma n\&view=download\&category slug=publicaciones-tecnicasinvestigacion-

5602 \&alias $=40356$ -

prevencion-control-

infecciones-asociadas-a-aten

4. Arango Díaz A, López Berrío $S$, Vera Núñez D, Castellanos Sánchez E, Rodríguez Sanabria PH, Rodríguez Feitó MB. Epidemiología de las infecciones asociadas a la asistencia sanitaria. Acta Méd Centro. 2018 [citado 20/06/2021]; 12(3):262-272. Disponible en: http://www.revactamedicacen tro.sld.cu/index.php/amc/articl e/view/923

5. Gonçalves Meneguet $M$, Marin da Silva Canini SR, Bellissimo-Rodrigues $\mathrm{F}$, Laus AM. Evaluación de los Programas de Control de Infección Hospitalaria en servicios de salud. Rev. Latino-Am. Enfermagem [Internet]. 2015 [citado 24 febrero 2021]; ene.-feb. 2015;23(1)(DOI:

10.1590/0104-

1169.0113.2530):98-105.

Disponible en:

https://www.scielo.br/pdf/rlae/ v23n1/es_0104-1169-rlae-2301-00098.pdf

6. Arista-Olvera Nayeli Xendali, Lozano-Nuevo José Juan, García-López Víctor Hugo, Narváez-Rivera Jorge Luis, Garro-Almendaro Ana Karen, Zamora-Cervantes, Laura Elena et al. Infección nosocomial por Acinetobacter y su efecto en un hospital de segundo nivel. Med. interna Méx. [revista en la Internet]. 2019 Ago [citado 2021 Ago 17] ; 35( 4 ): 477-484. Disponible en: http://www.scielo.org.mx/sciel o.php?script=sci_arttext\&pid= S0186-

$48662019000400477 \&$ Ing $=\mathrm{es}$

7. Subsecretaria de redes asistenciales. Informe de vigilancia IASS 2018 [Internet]. Santiago de Chile: Departamento de calidad y seguridad de la atención, Programa de control de IAAS ; 2018 [citado 16 mayo 2021] pp. 39-45. Disponible en: https://www.minsal.cl/wpcontent/uploads/2020/08/INF ORME-DE-VIGILANCIA-DEIAAS-2018.pdf

8. Rodríguez Álvarez Victoria Maritza, Hernández Seara Alejandro. Infecciones asociadas a la atención sanitaria en el Instituto Nacional de Angiología y Cirugía Vascular. Rev Cubana Angiol Cir Vasc 
[Internet]. 2021 Ago [citado 2021 Ago 17] ; 22( 2 ): e275. Disponible en: http://scielo.sld.cu/scielo.php? script=sci_arttext\&pid=S1682

$00372021000200005 \& \operatorname{lng}=\mathrm{es}$

9. Ministerio de Salud Pública. MSP. Normas de prevención y control de las infecciones nosocomiales. Ecuador.2018 [citado 2021 Feb 24]. Disponible en: https://www.salud.gob.ec/wpcontent/uploads/2019/10/Gac eta-IAAS-2018-

CORRECCIONESSNVSPV2.pdf

10. Yunga Quimí CHA, Pizarro Loor YL, Quimí Ramos LF. Factores predisponentes que conllevan a los pacientes a una neumonía asociada ventilación mecánica de la unidad de cuidados intensivos del Hospital Teodoro Maldonado Carbo. Periodo 2018 - 2019.Rev Mas vita[Internet]. 2020 [citado 16 Ago 2021]; 2(3):[aprox. 0 p.]. Disponible en: https://acvenisproh.com/revist as/index.php/masvita/article/v iew/101

11. Maguiña Vargas Ciro. Infecciones nosocomiales. Acta méd. Peru [Internet]. 2016 Jul [citado 2021 Oct 22] ; 33( 3 ): 175-177. Disponible en: http://www.scielo.org.pe/sciel o.php?script=sci_arttext\&pid= S1728-

$59172016000300001 \& \operatorname{lng}=\mathrm{es}$

12. Serra Valdés Miguel Ángel. La resistencia microbiana en el contexto actual y la importancia del conocimiento y aplicación en la política antimicrobiana. Rev haban cienc méd [Internet]. 2017 Jun [citado 2021 Oct 22] ; 16( 3 ): 402-419. Disponible en:

http://scielo.sld.cu/scielo.php? script=sci_arttext\&pid=S1729

$519 \times 2017000300011 \& \operatorname{lng}=\mathrm{es}$

13. Roque-Roque Joel Sack, Pereira-Victorio Cesar Johan. Ventilación e intubación respiratoria como factores de riesgo para neumonía en un hospital del tercer nivel. Rev Cubana Med Trop [Internet]. 2020 Dic [citado 2021 Oct 24] ; 72 ( 3 ): e478. Disponible en:

http://scielo.sld.cu/scielo.php? script=sci_arttext\&pid=S0375

$07602020000300008 \&$ Ing $=\mathrm{es}$ . Epub 08-Feb-2021

14. Tamayo-Pérez R, BlancoPedroso L, HernándezFerrales Y, Martínez-Utria A, De-la-Rosa-Frómeta J. Infecciones asociadas a la asistencia sanitaria en la unidad de cuidados intensivos de un hospital general. 
Revista Electrónica Dr. Zoilo E. Marinello Vidaurreta [Internet]. 2021 [citado 24 Oct 2021]; 46 (1) Disponible en: http://revzoilomarinello.sld.cu/ index.php/zmv/article/view/25 65

15. Céspedes Floirian Enrique, Borrego Fornaris Dámaris Lisset, Polanco Chong Ernesto Gustavo, Juy Aguirre Elisa, Rodríguez Sugve Lisbety. Neumonía asociada a la ventilación mecánica en niños y adolescentes. MEDISAN [Internet]. 2021 Abr [citado 2021 Oct 24] ; 25( 2 ): 319-331. Disponible en:

http://scielo.sld.cu/scielo.php? script=sci_arttext\&pid=S1029 30192021000200319\&lng=es . Epub 31-Mar-2021.

16. Chong YP, Bae I-G, Lee S-R, Chung J-W, Jun J-B, Choo EJ, et al. Clinical and Economic Consequences of Failure of Initial Antibiotic Therapy for Patients with CommunityOnset Complicated IntraAbdominal Infections. PLoS ONE [revista en internet]. 2015 [citado 24 Oct 2021]; 10(4): 1-11. Disponible en: https://journals.plos.org/ploso ne/article?id=10.1371/journal. pone.0119956.

17. Pezo Galdea MA, Menoscal Tómala KL, García Barreto A. Neumonía asociada a ventilación mecánica en pacientes ingresados en UCl: Etiología y factores de riesgo. RECIMUNDO. 2018 [citado 2021 Oct 24]; 2 (3). Disponible en: https://www.recimundo.com/i ndex.php/es/article/view/287/ pdf

18. Hernández P Glenn, Dalmazzo A Roberto, Gabriela De la Cerda S, Saavedra M Carmen, Calvo A Mario. Prevención de la neumonía asociada a ventilación mecánica. Rev. chil. infectol. [Internet]. 2001 [citado 2021 Oct 24]; 18(Suppl 2): 66-76. Disponible en: http://www.scielo.cl/scielo.php ?script=sci_arttext\&pid=S071 6$10182001018200004 \& \operatorname{lng}=\mathrm{es}$ http://dx.doi.org/10.4067/S07 16-10182001018200004 